\title{
Performance Enhancement for Outdoor Cooperative Localization Considering of Error Correlation between GPS data
}

\author{
Sanghyun Joo, Jihong Lee, Jiyong Kim and Joongki Park
}

\begin{abstract}
This paper presents an effective scheme to improve position estimation accuracy for multiple robot localization under outdoors. This scheme is applicable to cooperative localization (CL) system in which a robot exchanges no more relative position information between any of the robots. Applying the relative distance acquired by considering the error correlation of global positioning system (GPS) to cooperative localization framework, we can increase the positioning accuracy more than the CL system under certain circumstances without detecting other robot in a group. We consider a group of three robots equipped proprioceptive sensors such as odometer and exteroceptive sensors such as GPS and digital magnetic compass (DMC). In addition, each robot in the group is able to share the position data from sensors with others through wireless communication device. We prove to be strong error correlation between two robots' GPS position data acquired from experiment. Finally, in order to show the validity of our scheme, the proposed algorithm is implemented for multi-robot localization using experimental data of GPS error correlation.
\end{abstract}

Index Terms - cooperative localization, extended Kalman Filter, GPS error correlation, mobile robot

\section{INTRODUCTION}

In robotic application fields, mobile robot needs to know its exact position and orientation (pose) in order to carry out prescribed tasks. The problem of mobile robot localization has been a widely studied research topic in the last decade. There have been numerous approaches to the localization problem utilizing different types of sensors and a variety of methods from very simple one to the most complex and sophisticated. The main concept behind most of the current localization

Manuscript received November 14, 2008. This work was partly supported by the Dual-Use Technology Program of DAPA/DUTC and MIC/IITA [06-DU-LC-01, Development of Multi-Purpose Dog-Horse Robot based on the Network].

Sanghyun Joo is with the Control and Actuation System Group, Agency for Defense Development, Yuseong P.O. Box 35, Taejeon, 305600 Korea (phone: 82-42-821-4435; fax: 82-42-823-3400; e-mail: jooshce@ paran.com).

Jihong Lee and Jiyong Kim are with the BK21Mechatronics Group, Chungnam National University, Yuseong, Taejeon, 305764 Korea (e-mail: jihong@cnu.ac.kr, jiyong@cnu.ac.kr).

Joongki Park is with the Intelligent Robot Research Division, Electronics and Telecommunications Research Insititute, Yuseong, Taejeon, 305764 Korea (e-mail: jkp@etri.re.kr). schemes is to optimally combine measurements from proprioceptive sensors that monitor the motion of the robot with information collected by exteroceptive sensors that provide a representation of the environment and its signals. In recent years, multiple robot localization problems have been received tremendous interest from roboticists. The simplest solution of the multiple robot localization is to localize every robot independently, which is called self-localization (SL). But if the robots can detect each other robots and communicate between them, an optimal combing all the data coming from the sensors and some useful information transmitted by the other robots would improve the capability of localization of the robots, which is called cooperative localization (CL). In those applications, additional sensors such as sonars, laser scanners and cameras can be used for measuring other mobile robots' pose in order to increase the accuracy of localization([1]-[5]). The main limitations of such approaches are that the robots have to move in such a way that they can maintain the sensorial contact in order to measure the position of other robots. But it is impossible to maintain their distance to keep the sensorial contact all the time under outdoor environment.

In case that no relative position information among the robots in the group is available anymore, $\mathrm{CL}$ becomes equal to SL. Under the condition, we propose a simple method for outdoor multi-robot localization to increase the positioning accuracy using the error correlation of the position information from global positioning system (GPS). Specifically, we apply this method to a group of three robots equipped wheel encoder, digital magnetic compass (DMC), GPS receiver without the device to measure relative position. In addition, each robot in the group is able to share the position data from sensors with others through wireless communication device.

In the following section, we formulate more precisely the multi-robot localization problem we address. In section III, we set up two GPS receivers to acquire the position information of GPS receivers and compute the probability density functions (PDFs) of the latitude and the longitude distance of two GPS positions and calculate the mean and standard variation of the PDFs in order to find out the error correlation between GPS data. In section IV, through carrying out simulation, we will show how much improvement is the accuracy of the position estimation utilizing the suggested method for the multi-robot system under outdoor environment. 


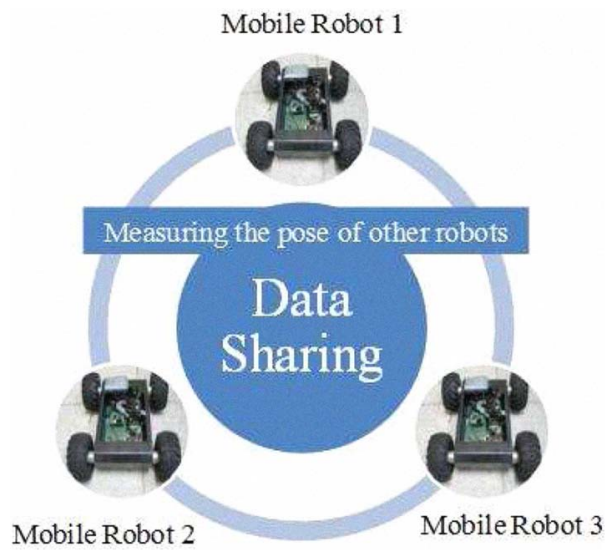

Fig. 1. Multiple robot system for cooperative localization

\section{Problem Statement}

In order to examine the effect of the error correlation of GPS data in multirobot localization problem, we state the following assumptions. First, we will deal with a group of three mobile robots under outdoor environment with flat terrain as depicted in Fig 1. In this group, each robot can move in a 2-dimentional space and it can change the heading of direction by differential-drive motion of wheels. Second, each robot carries proprioceptive and exteroceptive sensing devices in order to propagate and update its own position estimate. Specificially, each robot has odometric sensors which can count the rotations of the robot wheels and use this number to calculate the distance covered by the robot. And each robot has DMC which can measure the heading angle and GPS receiver which can obtain the absolute position information. Third, all the robots are equipped with communication devices (Bluetooth) that allow exchange of information within the group.

In this section, we introduce a mathematical model of a mobile robot and the Extended Kalman filter (EKF) equations to be applied to our problem.

\section{A. Kinematic Model}

Let us consider a differential-drive mobile robot as sketched in Fig.2. At discrete-time instants $k$ ( $k$ th time sample), the position of the robot is expressed by $\left(x_{k}, y_{k}\right)$ in global coordinate and the heading angle of the robot is written by $\theta_{k}$. The distance between the left and right wheel is expressed by $e$. The motion of the left and right wheels at time-instant $k$ is characterized by the angular velocities $\omega_{r, k}$ and $\omega_{l, k}$ respectively. We assume the radius $r$ of the left wheel is identical to the right one. If both pairs are driven forward with the same speed, then the robot move forward, but if they are driven in opposite directions, the robot will turn in place. And the wheel pairs may also be rotated at different speeds in same direction thereby enabling the robot to make gradual turns as it traverse. Actually, the command signals to control the robots are angular velocity signals of the wheel pairs. For simplicity, we consider the control signals

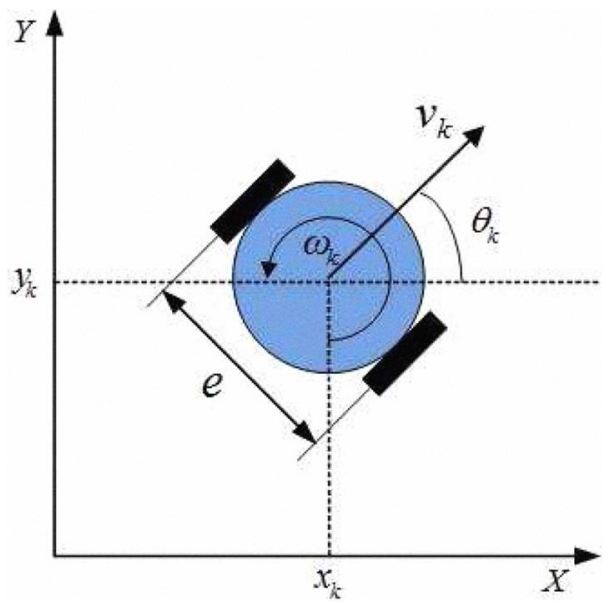

Fig. 2. Top veiw sketch of a differential-drive mobile robot

applied to the robot are

$$
u_{k}=\left[\begin{array}{ll}
V_{k} & \omega_{k}
\end{array}\right]
$$

where the linear velocity $V_{k}$ and angular velocity $\omega_{k}$ of the robot at time-instant $k$ are related to the angular velocity of the wheels by the following equation:

$$
\left\{\begin{array}{l}
V_{k}=\frac{r\left(\omega_{r, k}+\omega_{l, k}\right)}{2} \\
\omega_{k}=\frac{r\left(\omega_{r, k}-\omega_{l, k}\right)}{e}
\end{array}\right.
$$

The nominal (noise free) discrete process model equations of a mobile robot can be expressed as in [6]:

$$
\left\{\begin{array}{c}
x_{k+1}=x_{k}+T V_{k} \cdot \cos \left(\theta_{k}+T \omega_{k} / 2\right) \\
y_{k+1}=y_{k}+T V_{k} \cdot \sin \left(\theta_{k}+T \omega_{k} / 2\right) \\
\theta_{k+1}=\theta_{k}+T \omega_{k}
\end{array}\right.
$$

where $T$ is the sampling period.

The errors due to the control signals $\delta w_{k}=\left[\begin{array}{ll}\delta V_{k} & \delta \omega_{k}\end{array}\right]$ is supposed to be zero-mean, uncorrelated and Gaussian with constant variances $\sigma_{V}^{2}$ and $\sigma_{\omega}^{2}$. They are additive about the means $\bar{V}_{k}$ and $\bar{\omega}_{k}$ :

$$
\left\{\begin{array}{l}
V_{k}=\bar{V}_{k}+\delta V_{k} \\
\omega_{k}=\bar{\omega}_{k}+\delta \omega_{k}
\end{array}\right.
$$

Kalman filter estimation cycle can be divided into two steps ([7]): (a) The Prediction step where knowledge about the state of the system is propagated to the next step based on the assumptions about the evolution of the system equations, the measured control input, and the statistical description of the 
system noise, and (b) The Update step where measurement data are used to update the propagated estimates calculated during the previous step.

\section{B. Prediction}

In this section, we show the state prediction equations for the Kalman filter using the velocity measurements from the odometric sensors.

The state prediction takes place according to (3) as follows:

$$
X_{k \mid k-1}^{i}=X_{k-1 \mid k-1}^{i}+T\left[\begin{array}{c}
V_{k} \cos \left(\theta_{k-1 \mid k-1}+T \omega_{k} / 2\right) \\
V_{k} \sin \left(\theta_{k-1 \mid k-1}+T \omega_{k} / 2\right) \\
\omega_{k}
\end{array}\right]^{i}
$$

where

$$
\text { for robot } i, i=1,2,3
$$

$$
X_{k}^{i}=\left[\begin{array}{lll}
x_{k} & y_{k} & \theta_{k}
\end{array}\right]^{T}
$$

The prediction covariance is given by:

$$
P_{k \mid k-1}=\nabla f_{x, k} P_{k-1 \mid k-1} \nabla f_{x, k}^{T}+\nabla f_{w, k} Q_{k} \nabla f_{w, k}^{T}
$$

where $\nabla f_{x}$ represents the Jacobean with respect to the states, $\nabla f_{w}$ is the Jacobean with respect to the error sources and $Q_{k}$ is the noise strength matrix given by:

$$
\begin{gathered}
\nabla f_{x, k}=\left[\begin{array}{ccc}
\nabla f_{x, k}^{1} & 0_{3} & 0_{3} \\
0_{3} & \nabla f_{x, k}^{2} & 0_{3} \\
0_{3} & 0_{3} & \nabla f_{x, k}^{3}
\end{array}\right] \\
\nabla f_{w, k}=\left[\begin{array}{ccc}
\nabla f_{w, k}^{1} & 0_{3} & 0_{3} \\
0_{3} & \nabla f_{w, k}^{2} & 0_{3} \\
0_{3} & 0_{3} & \nabla f_{w, k}^{3}
\end{array}\right] \\
Q_{k}=\left[\begin{array}{ccc}
Q_{k}^{1} & 0_{2} & 0_{2} \\
0_{2} & Q_{k}^{2} & 0_{2} \\
0_{2} & 0_{2} & Q_{k}^{3}
\end{array}\right]
\end{gathered}
$$

with

$$
\begin{gathered}
\nabla f_{x, k}^{i}=\left[\begin{array}{ccc}
1 & 0 & -T V_{k} \sin \left(\theta_{k-1 \mid k-1}+T \omega_{k} / 2\right) \\
0 & 1 & T V_{k} \cos \left(\theta_{k-1 \mid k-1}+T \omega_{k} / 2\right) \\
0 & 0 & 1
\end{array}\right]^{i}, \\
\nabla f_{w, k}^{i}=\left[\begin{array}{cc}
T \cos \left(\theta_{k-1 \mid k-1}+T \omega_{k} / 2\right) & 0 \\
T \sin \left(\theta_{k-1 \mid k-1}+T \omega_{k} / 2\right) & 0 \\
0 & T
\end{array}\right]^{i}, \\
Q_{k}^{i}=\left[\begin{array}{cc}
\sigma_{V}^{2} & 0 \\
0 & \sigma_{\omega}^{2}
\end{array}\right]^{i}, 0_{3}=\left[\begin{array}{lll}
0 & 0 & 0 \\
0 & 0 & 0 \\
0 & 0 & 0
\end{array}\right], 0_{2}=\left[\begin{array}{ll}
0 & 0 \\
0 & 0
\end{array}\right] .
\end{gathered}
$$

\section{Update}

Let us assume that we are able to measure the each robot pose from GPS and DMC sensor at all time. In this case, we can also acquire the relative distance data from GPS data. They will be proven to have less variance compared to GPS position data of each robot in section III. We will apply the relative distance to the cooperative localization framework and we call this method the proposed CL in the following section.

The equation that connects the position and the measurement $Z_{k}$ (noisy absolute position) is as below:

$$
Z_{k}=H_{k} X_{k}+v_{k}
$$

where $H_{k}$ will be used in the Kalman filter model and it is defined as:

$$
H_{k}=\left[\begin{array}{ccc}
I_{3} & 0_{3} & 0_{3} \\
0_{3} & I_{3} & 0_{3} \\
0_{3} & 0_{3} & I_{3} \\
-I_{3} & I_{3} & 0_{3} \\
0_{3} & -I_{3} & I_{3} \\
I_{3} & 0_{3} & -I_{3}
\end{array}\right]
$$

with

$$
I_{3}=\left[\begin{array}{lll}
1 & 0 & 0 \\
0 & 1 & 0 \\
0 & 0 & 1
\end{array}\right]
$$

and $v_{k}$ is the noise of the observations that it is assumed to be zero-mean uncorrelated and Gaussian with $\sigma_{g p s, x}^{2}, \sigma_{g p s, y}^{2}$, $\sigma_{d m c}^{2}$ where are the variance of GPS data in X direction, the variance of GPS data in Y direction, and the variance of DMC sensor data respectively. In experiment, we will adjust the X, Y direction to the latitude and longitude direction respectively.

The residual covariance is given by:

$$
S_{k}=H_{k} P_{k \mid k-1} H_{k}^{T}+R_{k}
$$

where

$$
\begin{gathered}
R_{k}=\left[\begin{array}{cccccc}
R_{k}^{1} & 0_{3} & 0_{3} & 0_{3} & 0_{3} & 0_{3} \\
0_{3} & R_{k}^{2} & 0_{3} & 0_{3} & 0_{3} & 0_{3} \\
0_{3} & 0_{3} & R_{k}^{3} & 0_{3} & 0_{3} & 0_{3} \\
0_{3} & 0_{3} & 0_{3} & R_{c o r r}^{1} & 0_{3} & 0_{3} \\
0_{3} & 0_{3} & 0_{3} & 0_{3} & R_{c o r r}^{2} & 0_{3} \\
0_{3} & 0_{3} & 0_{3} & 0_{3} & 0_{3} & R_{c o r r}^{3}
\end{array}\right], \\
R_{k}^{i}=\left[\begin{array}{ccc}
\sigma_{g p s, x}^{2} & 0 & 0 \\
0 & \sigma_{g p s, y}^{2} & 0 \\
0 & 0 & \sigma_{d m c}^{2}
\end{array}\right], \quad R_{c o r r}^{i}=\left[\begin{array}{ccc}
\sigma_{c o r r}^{2}, x & 0 & 0 \\
0 & \sigma_{c o r r, y}^{2} & 0 \\
0 & 0 & 0
\end{array}\right]
\end{gathered}
$$


with $\sigma_{c o r r, x}^{2}, \sigma_{c o r r, y}^{2}$ are variance of GPS errors between robots in $\mathrm{X}$ and $\mathrm{Y}$ direction respectively.

The Kalman gain $W_{k}$ for the update is given by:

$$
W_{k}=P_{k \mid k-1} H_{k}^{T} S_{k}^{-1}
$$

And then, the state estimate and covariance updates are performed as follow:

$$
\begin{aligned}
X_{k \mid k} & =X_{k \mid k-1}+W_{k}\left(z_{k}-H_{k} X_{k \mid k-1}\right) \\
P_{k \mid k} & =P_{k \mid k-1}-W_{k} S_{k} W_{k}^{T}
\end{aligned}
$$

\section{ERROR CORRELATION BETWEEN GPS POSITION DATA}

If the robot is outdoors, GPS or DGPS could be used to update measurement in localization. A GPS receiver calculates its position by carefully timing the signals sent by the GPS satellites high above the earth. The signals from GPS include lots of errors due to atmospheric conditions, ephemeris errors, clock drift, and multipath etc. When there are two or more GPS receivers in close space, GPS position error of each receiver has considerable correlation with the others ([8],[9]), which is the fundamental principle of DGPS. Therefore we can remove common mode noise on GPS data through calculating the

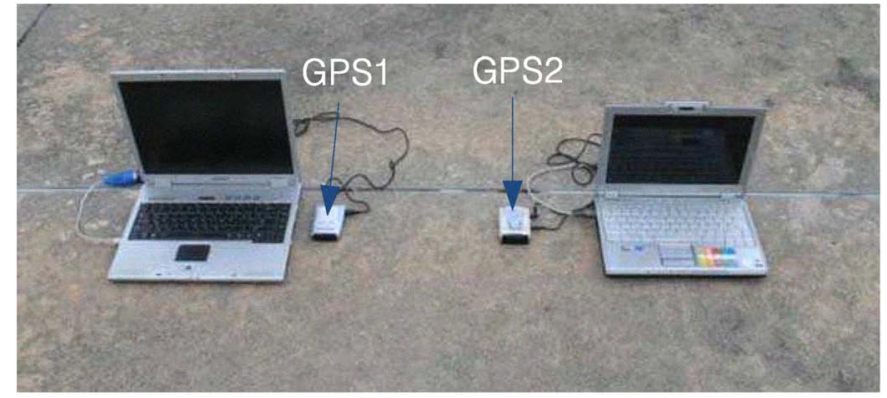

Fig. 3. Experimetal setup measuring correlaition between GPS data in common work space
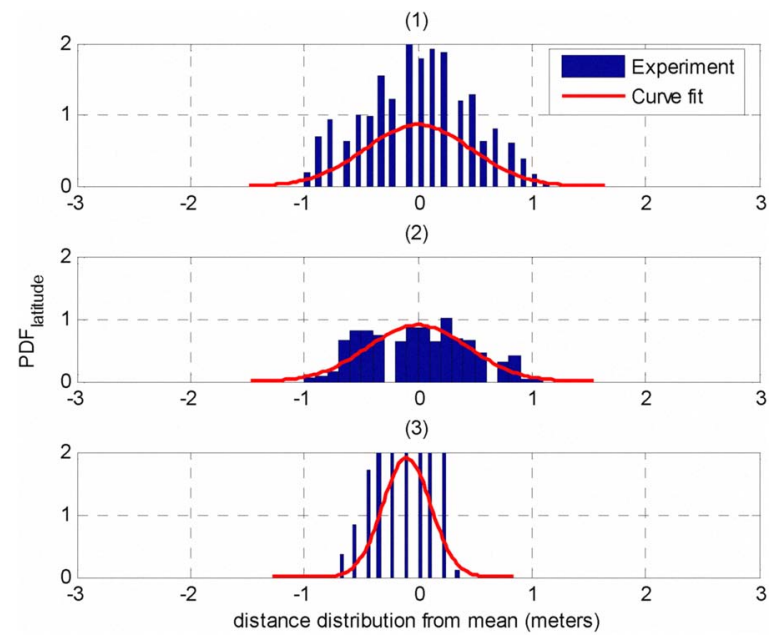

Fig. 4. PDFs of latitude distnace from (1) GPS1, (2) GPS2, (3) GPS1-GPS2
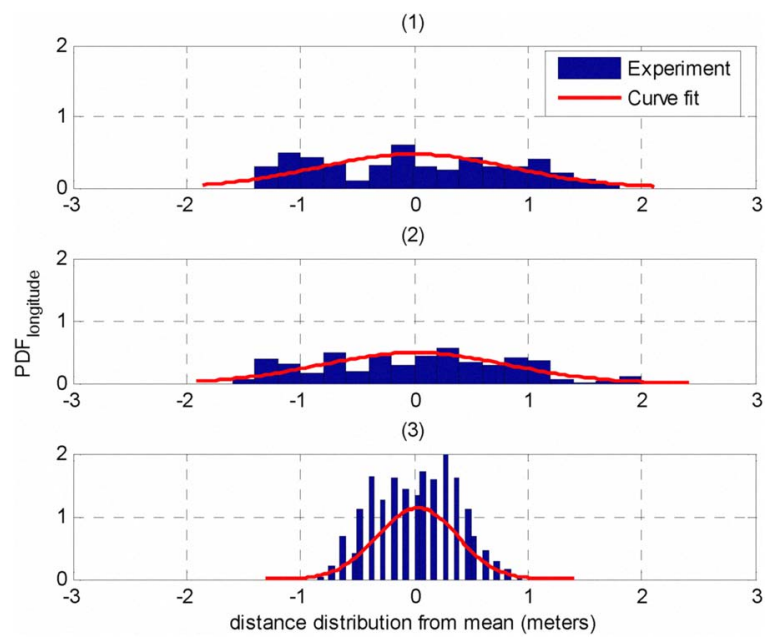

Fig. 5. PDFs of longitude distnace from (1) GPS1, (2) GPS2, (3) GPS1-GPS2

difference between GPS position data of the receivers.

In order to prove to be error correlation between GPS position data, we set up two GPS receivers in common work space (Fig. 3) and acquired the GPS position data during about 100 minutes. Fig. $4-5$ present the probability density functions (PDFs) of latitude and longitude distances from GPS1 and GPS2. From the calculated data, we can get some useful information of the error correlation between GPS position data shown as Table I

TABLE I

VALUE OF STANDARD DEVIATION FROM GPS DATA

\begin{tabular}{|c|c|c|}
\hline \multicolumn{2}{|c|}{ Standard Deviation of GPS } & $\begin{array}{c}\text { Value } \\
\text { [meter] }\end{array}$ \\
\hline \multirow{2}{*}{$\sigma_{g p s 1}$} & Latitude & 0.46 \\
\cline { 2 - 3 } & Longitude & 0.84 \\
\hline \multirow{2}{*}{$\sigma_{g p s 2}$} & Latitude & 0.44 \\
\cline { 2 - 3 } & Longitude & 0.82 \\
\hline \multirow{2}{*}{$\sigma_{g p s 1-g p s 2}$} & Latitude & 0.20 \\
\cline { 2 - 3 } & Longitude & 0.35 \\
\hline
\end{tabular}

\section{SimULATION RESULTS}

The proposed CL method was implemented and tested in simulation for the case of 3 mobile robots which are equipped wheel encoder, DMC, GPS receiver and wireless communication device to share the position data with others. Based on the real data acquired from the experiment (Section III), the main parameters used in simulation are determined as Table II. The simulated robot motion was recorded along with the dead reckoning (DR), GPS, SL and the proposed CL estimates. All measurements were available at $4 \mathrm{~Hz}$. And CL means the proposed CL in the following.

Fig. 6 presents the real paths of the robots (black solid lines), 
TABLE II

MAIN PARAMETERS USED IN SIMULATION

\begin{tabular}{|c|c|}
\hline Parameter & Value \\
\hline$\sigma_{g p s, x}$ & 0.45 meter \\
\hline$\sigma_{g p s, y}$ & 0.83 meter \\
\hline$\sigma_{d m c}$ & 0.0524 rad \\
\hline$\sigma_{c o r r, x}$ & 0.20 meter \\
\hline$\sigma_{c o r r, y}$ & 0.35 meter \\
\hline Operating Time & $50 \mathrm{sec}$ \\
\hline
\end{tabular}

the computed paths from the DR (blue dashed lines), the estimated paths of the robots' positions through the proposed CL (green cross marks). The 3 robots start from 3 different locations and they move at different speeds in common space. More specifically, one robot move in a straight line and the others gradually turn in a different curvature. In the case of DR, the position errors are increased as the robots move. But the results of CL show that they are similar to the real paths of the robots.

Fig. 7 shows the time history of the position errors of the each robot. It tell us CL is good estimator whose position error (black solid line) is small though the errors from GPS (red dots) and DR (blue cross marks)are big and changing with time. The results of SL are not expressed in the two figures before due to a little difference compared to CL. But in the following figures, difference between CL and SL can be recognized.

Fig. 8 depicts the comparison among the total cumulative position errors from DR, GPS, SL and CL for each robot. The total cumulative position error is the sum of errors which are computed at every sampling instant during operating time. From the results, we can know that the proposed CL is definitely superior to the others. As can be seen in Fig. 8, the

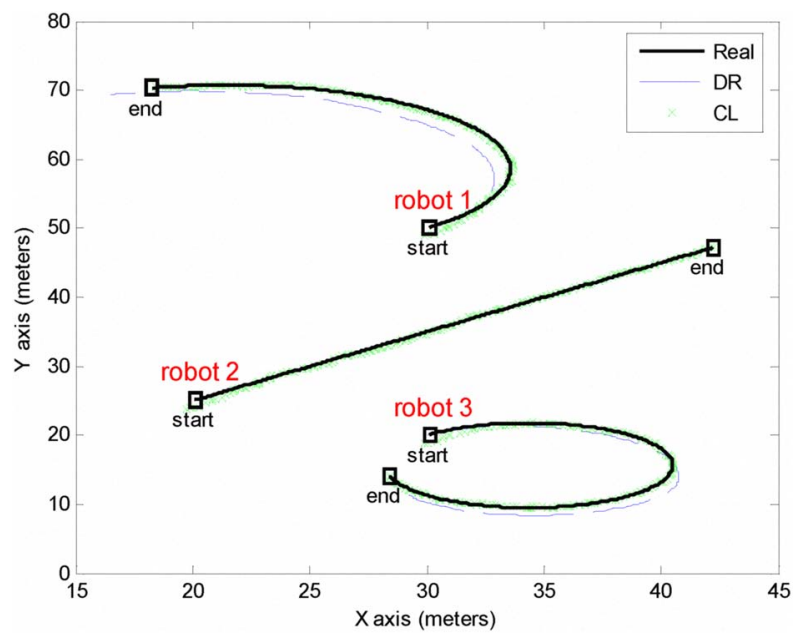

Fig. 6. Paths of 3 robots

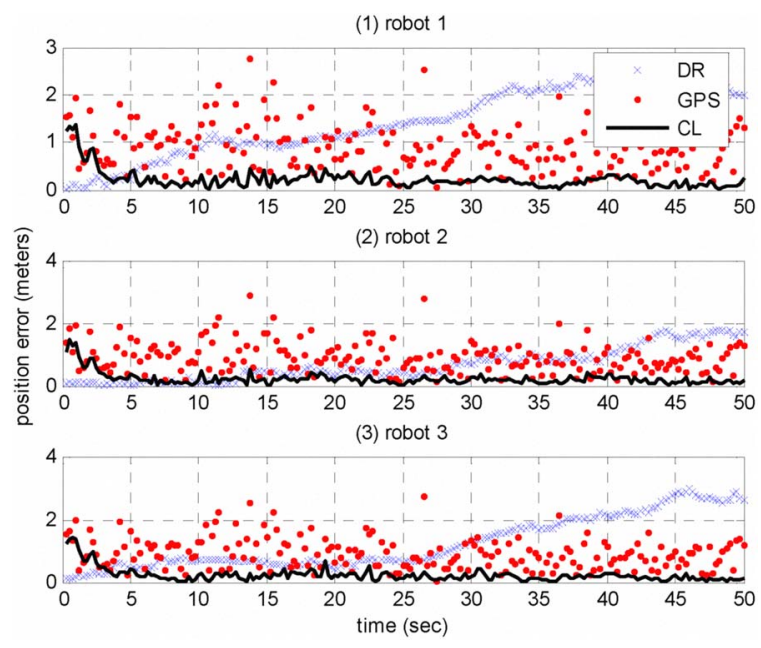

Fig. 7. Position errors of 3 robots

values of the cumulative position errors of DR have a bigger variation than the others, which is believed to be due to modeling error of odometer.

Fig. 9-11 illustrates the improvement of the estimation accuracy of the proposed CL. These show the comparison

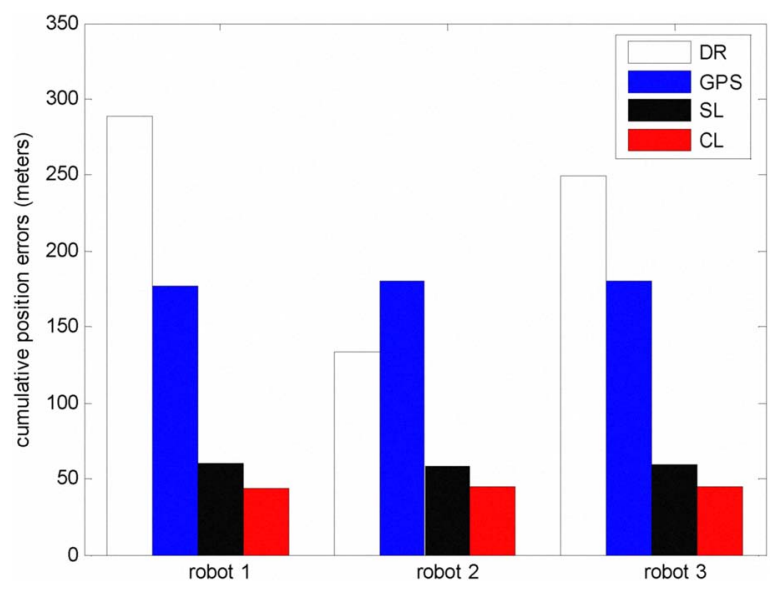

Fig. 8. Total cumulative position errors of 3 robots in one iteration

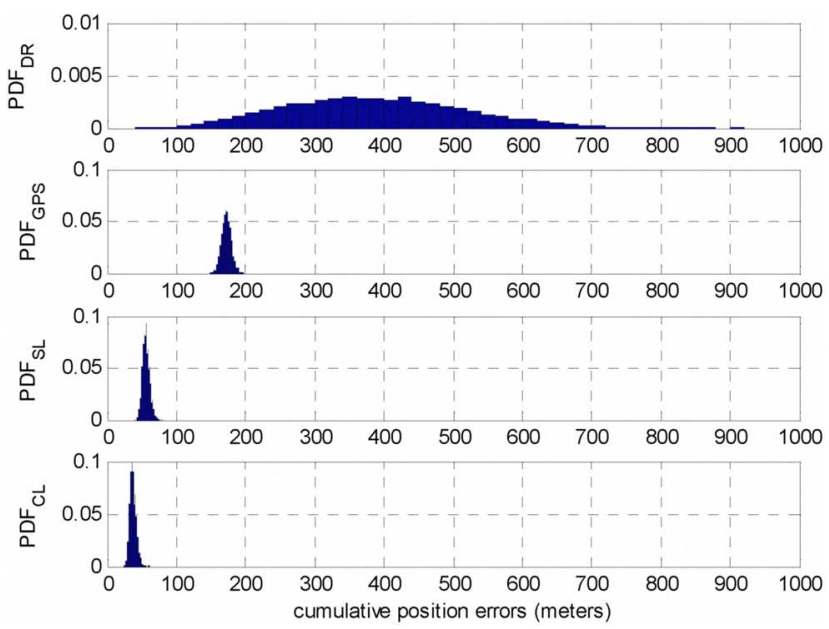

Fig. 9. PDFs of the total cumulative position error of the robot 1 

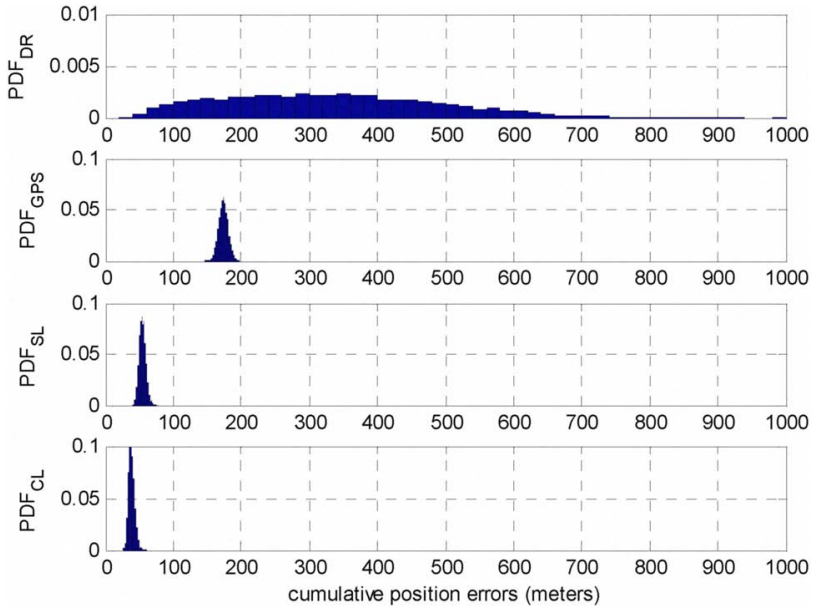

Fig. 10. PDFs of the total cumulative position error of the robot 2
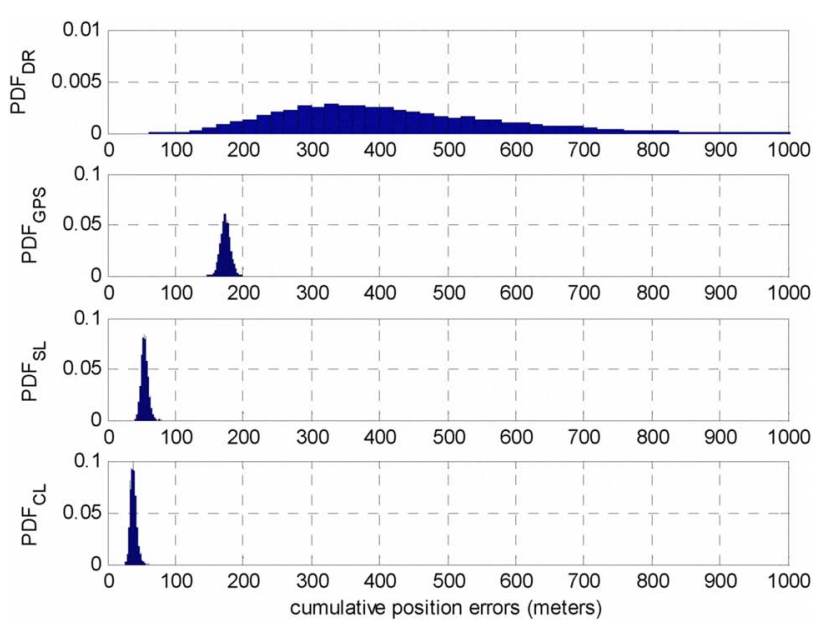

Fig. 11. PDFs of the total cumulative position error of the robot 3

among the PDFs of total cumulative position errors of DR, GPS, SL and CL for robot 1, 2 and 3 respectively. Each PDF was computed with a total of 10000 iterations. From these results,

TABLE I

MEAN AND STANDARD DEVIATION OF TOTAL CUMULATIVE POSITION ERRORS

\begin{tabular}{|l|c|c|c|}
\hline \multicolumn{2}{|c|}{} & $\begin{array}{c}\text { Mean } \\
\text { [meters] }\end{array}$ & $\begin{array}{c}\text { Standard Deviation } \\
\text { [meters] }\end{array}$ \\
\hline Robot 1 & SL & 56.0 & 5.0 \\
\cline { 2 - 4 } & CL & 37.0 & 4.5 \\
\hline Robot 2 & SL & 54.5 & 4.9 \\
\cline { 2 - 4 } & CL & 38.7 & 4.4 \\
\hline Robot 3 & SL & 54.8 & 5.0 \\
\cline { 2 - 4 } & CL & 38.4 & 4.5 \\
\hline
\end{tabular}

we can find out the quantities of the accuracy improvement for every robot as presented in Table III. It is worth noting that the performance of the localization method can be measured by the mean and the standard deviation of the cumulative position errors. The performance enhancement of the proposed CL algorithm can be measured by computing the rate of decrease in mean value of the total cumulative errors compared to them of SL. Therefore the improvement of the estimation accuracy is resulted as follows: (robot $1=33.9 \%$, robot $2=29.0 \%$, robot 3 $=29.9 \%$ ).

\section{CONCLUSION}

In this paper, we proposed an effective scheme for multiple robot localization in case that no relative position information among a team of robots under outdoor environment is available anymore. The proposed method used error correlation among GPS data to improve the positioning accuracy and was formulated to apply the relative distance information acquired from the GPS data to cooperative framework. The PDFs of the errors between the two GPS receivers were computed and they showed strong error correlation between GPS data. The proposed CL was successfully implemented using the error correlation data obtained from the experiment. Through some simulation work, it was shown that the proposed CL could increase the positioning accuracy more than SL. Finally, the evaluation method for the multiple robots localization was proposed by computing the PDFs of the total cumulative position errors.

In future work, we plan to manufacture mobile robots and carry out the outdoor experiment using the proposed scheme.

\section{REFERENCES}

[1] S. Roumeliots, and G. Bekey, "Distributed multirobot localization" IEEE Transl. on Robotics and Automation, vol. 18, no.5, pp. 781-795, October 2002.

[2] S. Roumeliots, and G. Bekey, "Collective localization: a distributed Kalman filter approch of groups of mobile robots", in Proc. 2000 IEEE Int. Conf. on Robotics and Automation, April 2000.

[3] R. Madhavan, K. Fregene, and L. E. Parker, "Distributed coopetative outdoor multirobot localization and mapping", Autonomous Robots 17, pp 23-39, 2004.

[4] A. J. Davison, and N. Kita, "Active visual localization for cooperating inspection robots", in Proc. 2000IEEE/RSJ Int. Conf. on Intelligent Robots and Systems, vol. 3, pp. 1709-1715, 2000.

[5] K. Kato, H. Ishiguro, and M. Barth, "Identifying and localizing robots in a multi-robot system environment", in Proc. IEEE Int. Conf. on Intelligents Robot and Systems, vol. 2, pp. 2896-2903, 1998

[6] G. Antonelli, S. Chiaverini, and G. Fusco, "A calibration method for odometry of mobile robots based on the least-squares technique: theory and experimental validation", IEEE Transl. on Robotics, vol. 21, no. 5, October 2005.

[7] P. S. Maybeck, Stochastic Models, Estimation and Control, vol. 141-1 of Mathematics in Science and Engineering, Academic Press, 1979.

[8] K. Jo, J. Lee, and J. Kim, "Cooperative multi-robot localization using differential position data", in Proc. 2007 IEEE/ASME Int. Conf. Advanced Intelligent Mechatronics, September 2007.

[9] K. Jo and J. Lee, "Multi-robot cooperative localization with optimally fused information of odometer and GPS", in Proc. 2007 Int. Conf. Control, Automation and Systems, pp. 601-605, October 2007. 